\title{
Basic science and better medicine
}

To the editor - Your news item on the recent research assessment of Britain's universities (Nature Medicine 3, 133; 1997) rightly drew attention to the high international standard of institutions in some particular subject areas of clinical and preclinical activity. However, the distinction that was then drawn between the ratings in medical sciences and those in basic sciences seemed to me to obscure rather than illuminate. As though biochemistry were not an essential component of medical research, you bemoan the number of institutions exhibiting excellence in this discipline.
Yet there must be no UK medical school without an embedded or associated biochemistry department. Indeed the pages of Nature Medicine provide monthly evidence of the interdependence of basic biomedical sciences and clinical medicine. It is almost axiomatic that excellence in clinical research can only flourish in the environment provided by excellent biomedical sciences. To prove the point, taking all the medical institutions entered in the recent research assessment exercise and aggregating their scores in all the biomedical units of assessment, one-third of the in- stitutions (10/30) have overall scores in the "international excellence" range (more than 5). These top ten institutions include seven that are federated to the University of London, as well as the Universities of Oxford, Cambridge and Edinburgh. (For the full table, see http:// www.icr.ac.uk/resserv/biomedRAE.html) A rather healthier state of affairs than your moribund diagnosis would suggest.

\section{KEITH SNELL}

Section of Structural Biology, Institute of Cancer Research, University of London, 15 Cotswold

Road, Sutton, Surrey SM2 5NG, UK

\section{Progress on blood substitutes}

To the editor - Your news article "Blood substitutes are gasping for air" (Nature Medicine 3, 10; 1997) summarizes the views of Col. John Hess, Chief of the US Army's Blood Research Detachment, who has concluded that the toxicities of blood substitute products will prevent them from reaching the market. In fact, optimism among scientists working in the field is very high, and two products (one initially developed by the US Army) are now in FDA-approved phase III clinical trials. Although much research in blood substitutes has been focused on their side effects, there is no recorded instance of serious injury resulting from their administration to humans.

Many studies (some carried out by the US Army) have not shown neurotoxicity in animals, and renal injury is eliminated when hemoglobin is properly crosslinked. Vasoactivity may be advantageous in some clinical settings such as shock and sepsis. The significance of hemoglobinendotoxin interaction is not known, and it is possible that it might actually facilitate endotoxin removal. The mild side effects of perfluorocarbon emulsions have been either eliminated by reformulation or are managed with common anti-inflammatory agents. The "stiff lung" problem seen with some PFC emulsions has been solved by adjustments to the formulation by at least two companies.

The US military, presumably influenced by Col. Hess's views, now supports little or no research in blood substitutes, despite the Naval Research Advisory Committee's recommendation that efforts be stepped up $^{1}$. The consequences for health care, particularly in areas of the world where blood services are not available, are enormous. It is hoped that responsible scientists will continue to chip away at the problems that remain in the development of therapeutic oxygen carriers.

\section{ROBERT M. WINSLOW}

Professor of Medicine, University of California, San Diego, V.A. Medical Center (111-E), 3350 La Jolla Village Drive, San Diego, CA 92161, USA

1. Naval Research Advisory Committee Report, 92-2. Delivery of Artificial Blood to the Military. [Office of the Assistant Secretary of the Navy (Research, Development and Acquisition), Washington, DC 20350-1000].

\section{Neuronal xenotransplantation in Parkinson's disease}

To the editor - Our study of surviving fetal pig dopamine neurons transplanted into a patient with Parkinson's disease was reported recently in Nature Medicine ${ }^{1}$.
A separate article describing pig-retrovirus infection of human cells in culture ${ }^{2}$ and a general commentary ${ }^{3}$ on risks of retroviruses in xenotransplantation appeared in the same issue. Since no specific comment was made to differentiate between these reports, a number of issues need to be clarified for the benefit of concerned patients, their families, and the scientific and medical community.

Foremost, the fetal pig cell trial for Parkinson's disease is indeed an open safety trial (phase I) approved and carefully monitored by the Food and Drug Administration (FDA) of the United States. Our report exclusively examined the neurohistology and survival, in a Parkinson patient, of extensively tested and screened normal fetal pig neural cells, whereas the article by Patience et al. focused on the virology of a laboratory-grown pig kidneycancer cell line, known to produce C-type retroviruses. The latter study showed that such viruses can infect human cell lines in cell-culture experiments. However, the Ctype retroviruses are not new and have previously not been shown to cause human disease, even though they can be activated and associated with malignant cells ${ }^{4-5}$. Also, Patience et al. referred to the DNA pieces found in the pig genome as pig endogenous retroviruses (PERV), when they may be better described as pig endogenous retroviral sequences (PERS). The press consequently misunderstood and reported that actual "viruses" exist in all pig cells (e.g., the Boston Globe, 1 March 1997), instead of sometimes transcribed but usually untranslated provirus DNA sequences typically seen in at least $1-10 \%$ of any normal mammalian genome ${ }^{3}$.

The FDA received a document (ref. 6) four months ago, reviewing extensively the current FDA guidelines and risks of microbe transmission in xenotransplanta- 